Research Article

\title{
Performance Analysis of Short Journal Bearing under Thin Film Lubrication
}

\author{
Sandeep Soni and D. P. Vakharia \\ Department of Mechanical Engineering, SVNIT, Surat 395007, India \\ Correspondence should be addressed to Sandeep Soni; sandytit2004@gmail.com
}

Received 26 February 2014; Accepted 30 March 2014; Published 24 April 2014

Academic Editors: W.-H. Chen and P. M. Mariano

Copyright (C) 2014 S. Soni and D. P. Vakharia. This is an open access article distributed under the Creative Commons Attribution License, which permits unrestricted use, distribution, and reproduction in any medium, provided the original work is properly cited.

\begin{abstract}
The steady state performance analysis of short circular journal bearing is conducted using the viscosity correction model under thin film lubrication conditions. The thickness of adsorbed molecular layers is the most critical factor in studying thin film lubrication, and is the most essential parameter that distinguishes thin film from thick film lubrication analysis. The interaction between the lubricant and the surface within a very narrow gap has been considered. The general Reynolds equation has been derived for calculating thin film lubrication parameters affecting the performance of short circular journal bearing. Investigation for the load carrying capacity, friction force, torque, and power loss for the short circular journal bearing under the consideration of adsorbed layer thickness $(2 \delta)$ has been carried out. The analysis is carried out for the short bearing approximation $(L / D=0.5)$ using Gumbel's boundary condition. It has been found that the steady state performance parameters are comparatively higher for short circular journal bearing under the consideration of adsorbed layer thickness than for plain circular journal bearing. The load carrying capability of adsorbed layer thickness considered bearing is observed to be high for the specified operating conditions. This work could promote the understanding and research for the mechanism of the nanoscale thin film lubrication.
\end{abstract}

\section{Introduction}

During the past few decades hydrodynamic journal bearings have received great attention from practical and analytical engineers. The rapid growth of journal bearing technology is mainly due to its wide range of engineering applications such as precision machine tools, high speed aircraft, nuclear reactors, and textile spindles. The development of thin film lubrication has provided a theoretical basis to reveal the practical engineering; the film is developed on the basis of the study in a microstate. At present, the calculation of molecular-level thin film has just begun.

The viscosity modifying model should have universal significance; it is natural that also applies to the journal bearing [1]. Viscosity, an important technical specification of a lubricant, is an important parameter for fluid lubrication analysis. When performing the analysis for a general bearing with fluid lubrication, the viscosity is usually kept constant [2]. But modifications are necessary in some conditions, for example, pressure-viscosity correction for high-pressure conditions and temperature $[2,3]$ for high-speed and heavy-load conditions. The lubrication of bearings is directly affected by the flow properties of the liquid lubricant. One key feature of lubricant is its viscosity distribution in the bearing. The distribution is dependent on the adsorbent properties of the surface and the velocity distribution of the lubricant [4].

Therefore, investigation of steady state behavior of a short journal bearing under thin film lubrication condition is necessary. This paper describes the behavior of a plain circular profiled journal bearing under the thin lubrication regime. In this paper the short circular journal bearing has been analyzed analytically taking consideration of the effect of adsorbed layer thickness $(2 \delta)$ on the various performance parameters at steady state condition. 


\section{Reynolds' Equation for Short Journal Bearing}

For steady-state and incompressible flow, the Reynolds equation in two-dimensional form for pressure generation is given as [5]

$$
\frac{\partial}{\partial x}\left(h^{3} \frac{\partial p}{\partial x}\right)+\frac{\partial}{\partial z}\left(h^{3} \frac{\partial p}{\partial z}\right)=6 U \mu \frac{d h}{d x}
$$

The short bearing approximationassumes a sufficiently short bearing in the axial direction and neglects the first term of the left-hand side of (1), allowing it to be solved analytically or numerically. The Reynolds equation for a short journal bearing is given as

$$
\frac{\partial}{\partial z}\left(h^{3} \frac{\partial p}{\partial z}\right)=6 U \mu \frac{d h}{d x}
$$

Equation (2) can be converted to nondimensional form and is used to solve the equation for pressure. Equation (2) is solved for plain circular journal bearing with required accuracy. For solving short circular journal bearing $(L / D=$ $0.5)$, Gumbel's boundary condition is used. At the two ends of the bearing, the pressure is equal to the atmospheric pressure; $P=0$. These boundary conditions can be written as $P=0$; $z=0$ to $( \pm L / 2)$ and $P=0 ; \theta=0,2 \pi$. The oil film thickness equation for plain circular journal bearing is given as [5]

$$
h_{\text {Circular }}=C *[1+\varepsilon \cos \theta] \text {. }
$$

The bearing geometry and coordinates considering adsorbed layer thickness for proposed journal bearing are shown in Figure 1.

The influence of the adsorbed layer thickness in the thin film lubrication to the lubricant viscosity is considered here, and assuming that two surfaces have the same adsorption characteristics $\left(\delta_{1}=\delta_{2}\right)$, the equivalent viscosity correction model is introduced in the analysis of plain circular journal bearing. Equation (4) gives the relationship between adsorbed layer thickness $(2 \delta)$ and oil film thickness for circular journal bearing [4]:

$$
\frac{\mu}{\mu_{0}}=\frac{h}{h-2 \delta} \text {. }
$$

Equation (2) has been solved taking (3) and (4) into consideration for the effect of thin film lubrication. The final modified form of generalized Reynolds' equation using short approximation with adsorbed layer thickness is given by

$$
\frac{\partial}{\partial z}\left(h^{2}\{h-2 \delta\} \frac{\partial p}{\partial z}\right)=\frac{6 U \mu_{0}}{R} \frac{d h}{d \theta} .
$$

\section{Computational Methodology}

The Reynolds equation (5) is expressed in terms of film thickness " $h$ ", pressure " $p$ ”, entraining velocity " $U$ ”, adsorbed layer

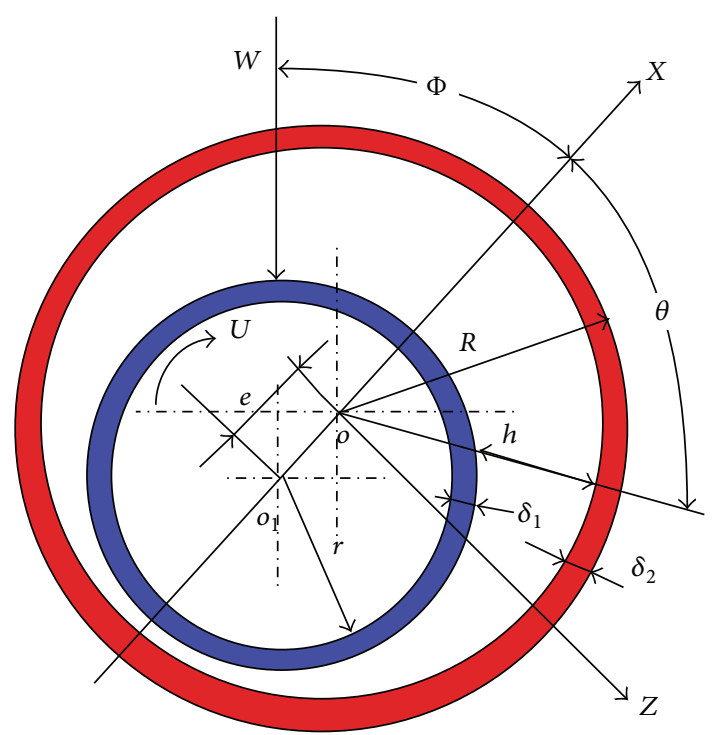

FIGURE 1: Short circular journal bearing considering adsorbed layer thickness.

thickness " $2 \delta$ " and dynamic viscosity " $\mu_{0}$ ". Nondimensional forms of the equation's variables are following:

$$
\begin{array}{cc}
x=R \theta ; \quad z=\left(\frac{L}{2}\right) z^{*} ; \quad \lambda=\left(\frac{D}{L}\right) ; \\
h=C h^{*} ; \quad p=\left(\frac{6 \mu U R}{C^{2}}\right) p^{*} ; \quad \delta=C \delta^{*} .
\end{array}
$$

The Reynolds equation (5) in its nondimensional form is expressed as

$$
\left(\frac{D}{L}\right)^{2}\left(h^{* 3}-2 \delta h^{*}\right)\left(\frac{\partial^{2} p^{*}}{\partial z^{* 2}}\right)=6 \frac{d h^{*}}{d \theta} .
$$

All terms in (7) are nondimensional apart from " $D$ ” and " $L$ " which are only present as a nondimensional ratio. Numerical solution of (7) has been carried out using finite difference method by approximating the unwrapped bearing surface as a rectangular grid and applying central differentiation to the governing equation to find expression of hydrodynamic pressure. For determining the pressure distribution and static performance characteristics of both plain circular short journal bearing and adsorbed layer thickness $(2 \delta)$ considered plain circular short journal bearing, a MATLAB program has been developed.

\section{Steady State Bearing Performance Parameters}

The solution of (7) for both plain circular short journal bearing and adsorbed layer thickness $(2 \delta)$ considered plain circular short journal bearing satisfying the boundary conditions and film thickness yields the pressure distribution from which load capacity, attitude angle, flow, friction force, and coefficient of friction are computed. These important bearing parameters are then modified by the consideration 

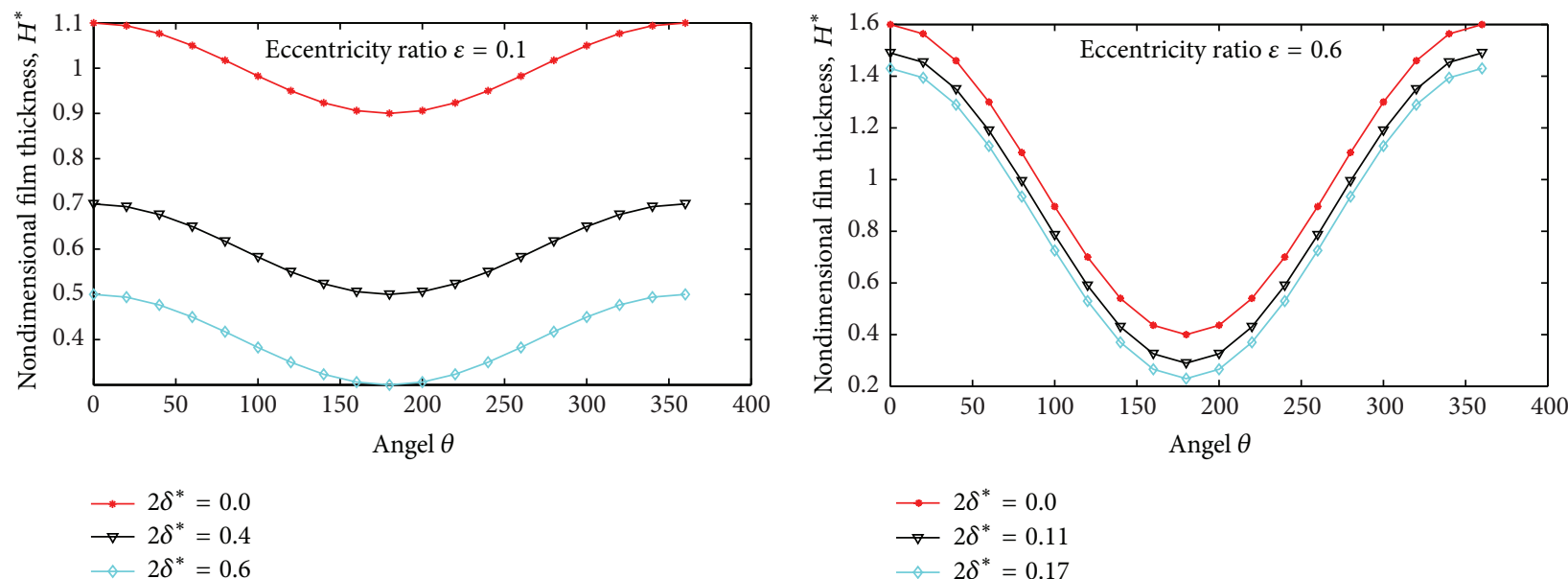

FIGURE 2: Nondimensional film thickness variation of short journal bearing at $\varepsilon=0.1$ and 0.6 for different values of $2 \delta^{*}$.
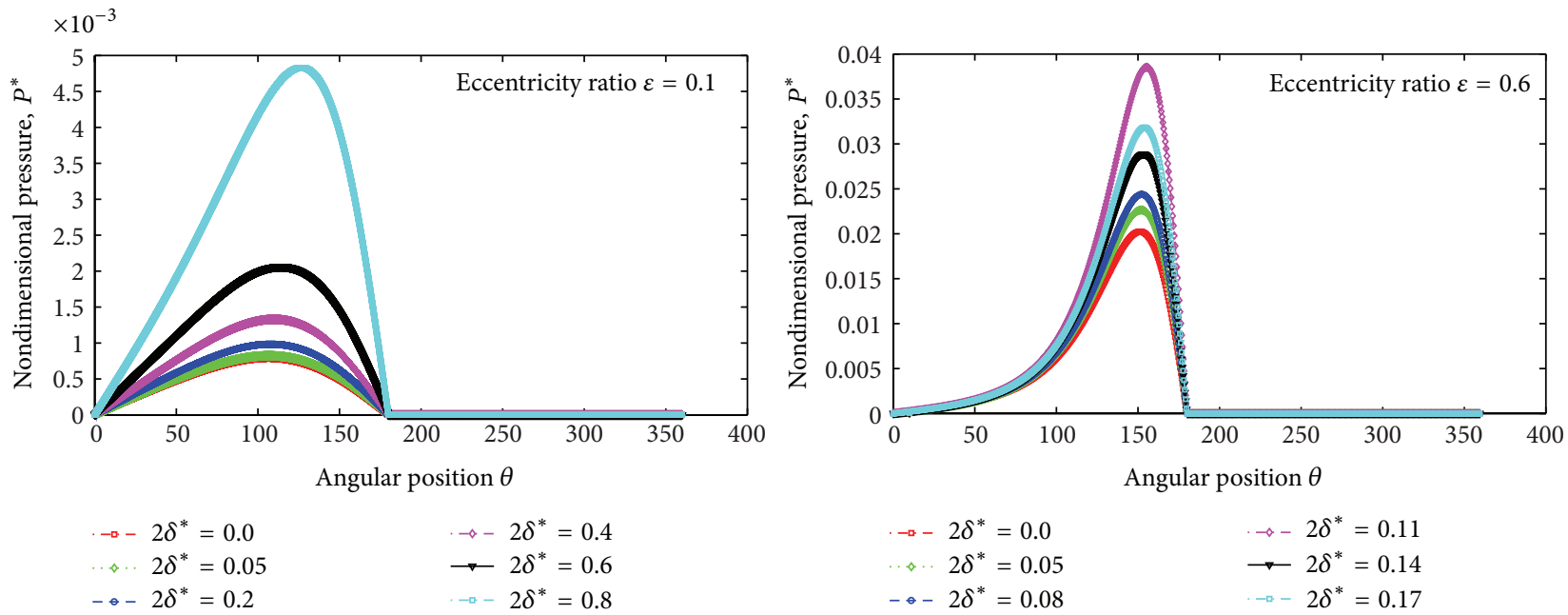

FIGURE 3: Nondimensional pressure distribution of short journal bearing at $\varepsilon=0.1$ and 0.6 for different values of $2 \delta^{*}$.
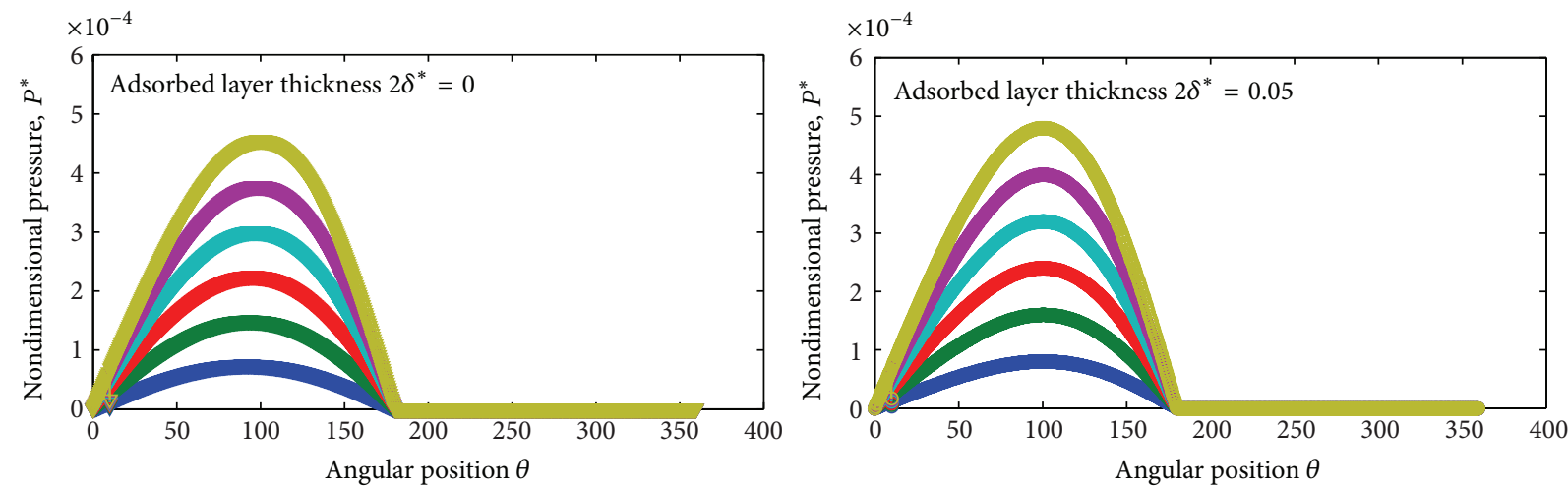

$$
\begin{array}{ll}
\nabla \varepsilon=0.01 & \nabla \varepsilon=0.04 \\
\nabla \varepsilon=0.02 & \nabla \varepsilon=0.05 \\
\nabla \varepsilon=0.03 & \nabla \varepsilon=0.06
\end{array}
$$$$
\begin{aligned}
& \multimap \varepsilon=0.01 \\
& \multimap \varepsilon=0.02 \\
& \multimap \varepsilon=0.03
\end{aligned}
$$$$
\multimap \varepsilon=0.04
$$$$
\varepsilon=0.05
$$$$
\multimap \varepsilon=0.06
$$

FIGURE 4: Nondimensional pressure distribution of short journal bearing at different values of $\varepsilon$ for $2 \delta^{*}=0.0$ and $2 \delta^{*}=0.05$. 

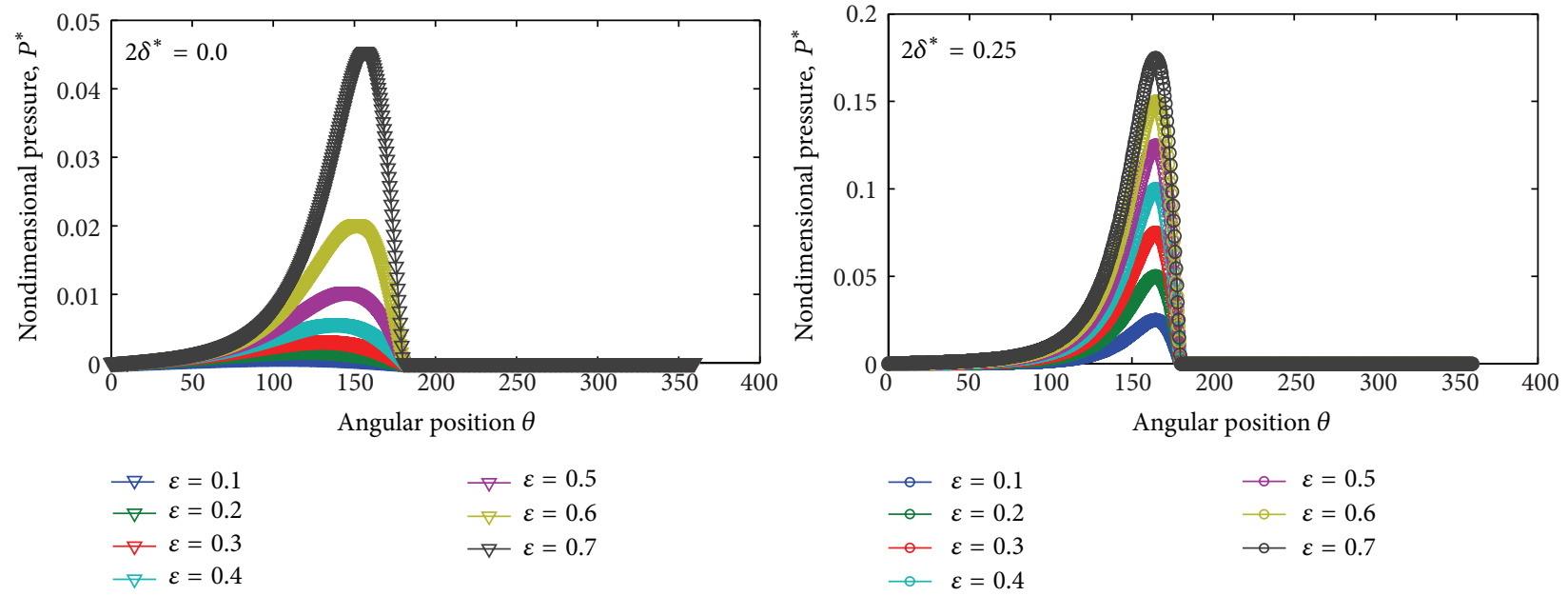

$$
\begin{aligned}
& \nabla \varepsilon=0.5 \\
& \nabla \varepsilon=0.6 \\
& \nabla \varepsilon=0.7
\end{aligned}
$$$$
\begin{aligned}
& \multimap \varepsilon=0.1 \\
& \multimap-\varepsilon=0.2 \\
& \multimap-\varepsilon=0.3 \\
& \multimap \quad \varepsilon=0.4
\end{aligned}
$$$$
\multimap \varepsilon=0.5
$$$$
\multimap \varepsilon=0.6
$$$$
\multimap \varepsilon=0.7
$$

FIGURE 5: Nondimensional pressure distribution of short journal bearing at different values of $\varepsilon$ for $2 \delta^{*}=0.0$ and $2 \delta^{*}=0.25$.
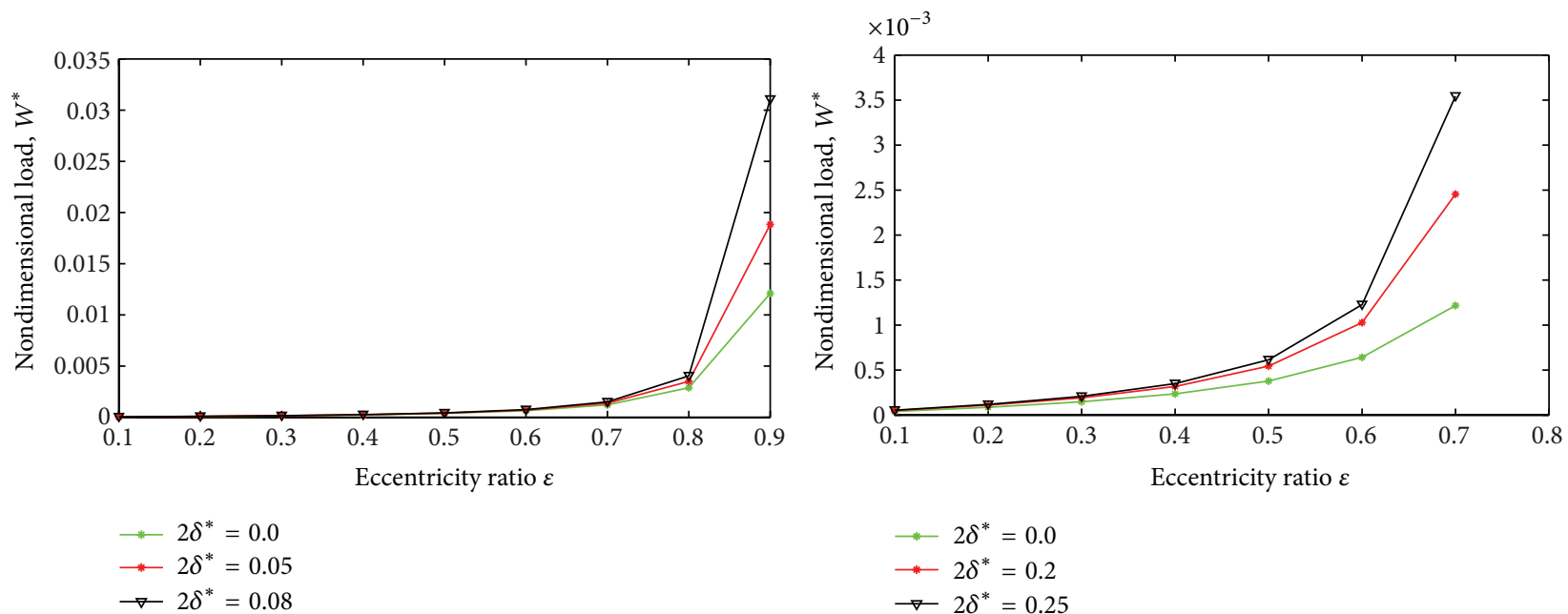

FIGURE 6: Nondimensional load capacity of short journal bearing as a function of the adsorbed layer thickness $\left(2 \delta^{*}\right)$.

of adsorbed layer thickness $(2 \delta)$. The film thickness and other parameters are calculated by (3) and (7), respectively.

4.1. Load Capacity. The following are the two equations for the integration for the load capacity components in the directions of $W_{x}$ and $W_{y}$ of the bearing centreline and the normal to it:

$$
\begin{gathered}
W_{x}=-2 \int_{0}^{\pi} \int_{0}^{L / 2} p \cos \theta R d \theta d z \\
W_{y}=2 \int_{0}^{\pi} \int_{0}^{L / 2} p \sin \theta R d \theta d z
\end{gathered}
$$

The load capacity components in the directions of $W_{x}$ and $W_{y}$ are modified by the consideration of adsorbed layer thickness $(2 \delta)$ for taking the effect of thin film lubrication on the performance of load carrying ability of proposed journal bearing. The two load components yield the resultant load capacity of the bearing, $W$ :

$$
W=\sqrt{W_{x}^{2}+W_{y}^{2}}
$$

The attitude angle, $\phi$, is determined from the two load components:

$$
\tan \phi=\left(\frac{W_{y}}{W_{x}}\right) .
$$

4.2. Frictional Properties. The friction force, $F_{f}$, is obtained by integration of the shear stress, $\tau$, over the complete surface area of the journal:

$$
F_{f}=\int_{A} \tau d A .
$$



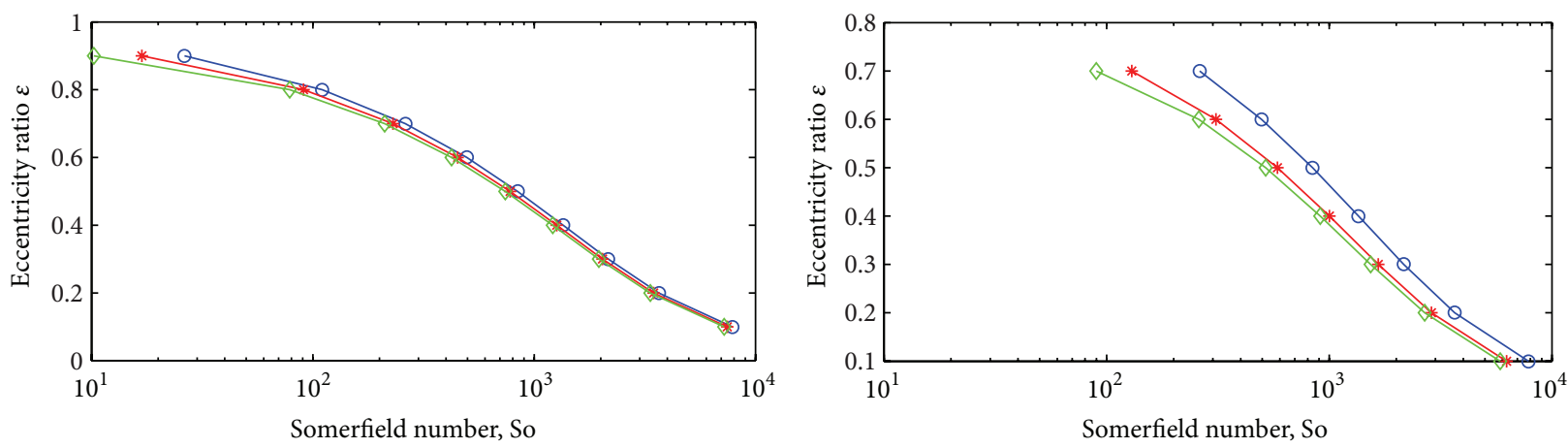

$$
\begin{aligned}
& \multimap 2 \delta^{*}=0.0 \\
& --2 \delta^{*}=0.05 \\
& \neg 2 \delta^{*}=0.08
\end{aligned}
$$

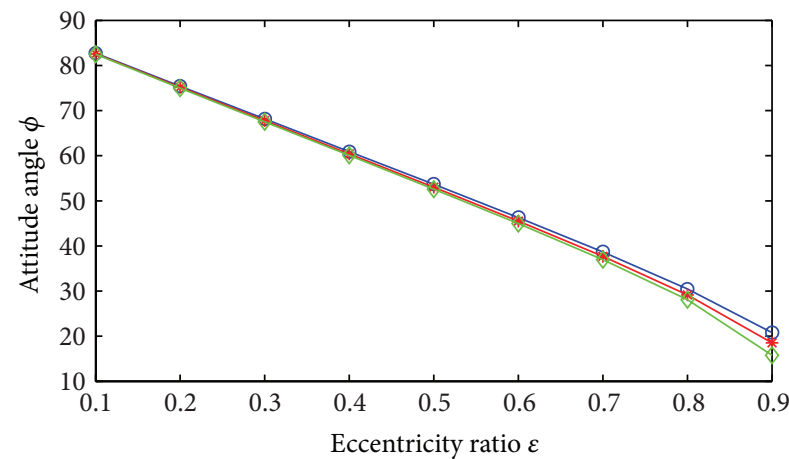

$\rightarrow 2 \delta^{*}=0.0$

$\because-2 \delta^{*}=0.2$

$$
\smile 2 \delta^{*}=0.25
$$

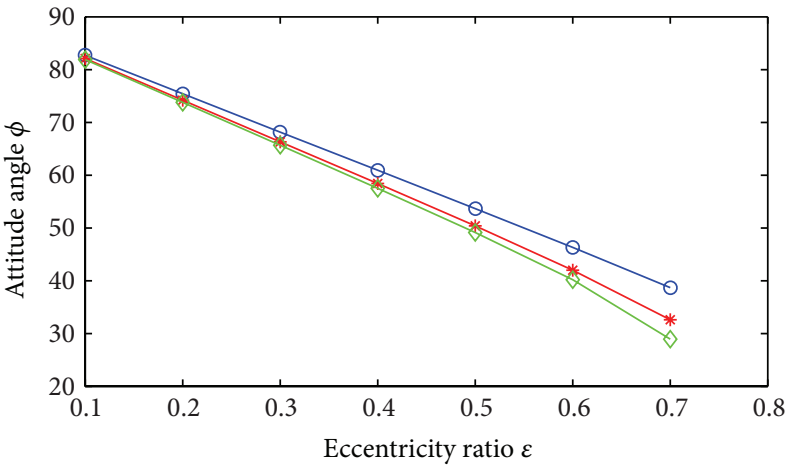

$$
\begin{aligned}
& \multimap 2 \delta^{*}=0.0 \\
& \rightarrow-2 \delta^{*}=0.05 \\
& \neg 2 \delta^{*}=0.08
\end{aligned}
$$

$$
\begin{aligned}
& \multimap 2 \delta^{*}=0.0 \\
& \rightarrow-2 \delta^{*}=0.2 \\
& \neg 2 \delta^{*}=0.25
\end{aligned}
$$

FIGURE 7: Somerfield number and attitude angle variation to eccentricity ratio at different values of adsorbed layer thickness $\left(2 \delta^{*}\right)$.
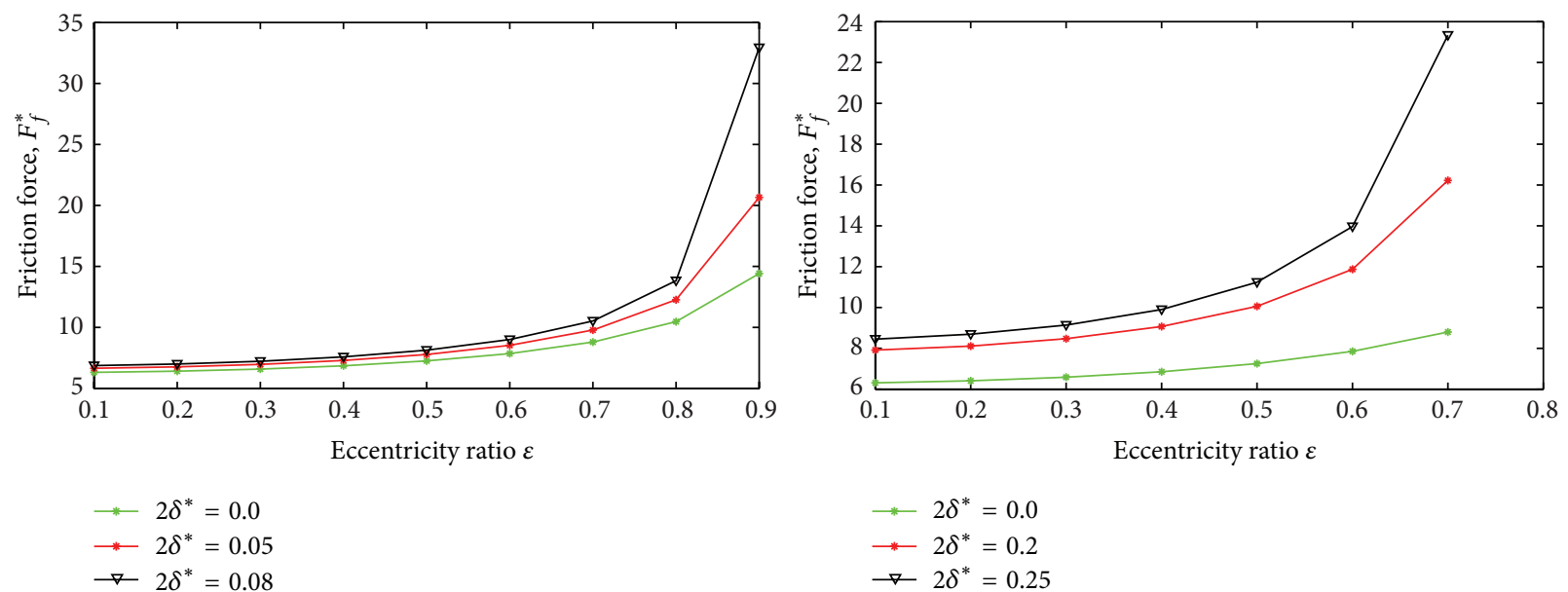

FIGURE 8: Nondimensional friction force of short journal bearing as a function of the adsorbed layer thickness $\left(2 \delta^{*}\right)$.

The frictional force further modified by the consideration of adsorbed layer thickness $(2 \delta)$ for taking the effect of thin film lubrication on the performance of load carrying ability of proposed journal bearing. The effect of adsorbed layer thickness $(2 \delta)$ on the frictional torque and energy-loss per unit time are also calculated.
4.3. Lubricant Flow Rate. The lubricant flux flow through the end bearing is as follows:

$$
Q_{z}=2 \int_{0}^{\pi}-\left(\frac{h^{3}}{12 \mu}\right)\left(\frac{d p}{d z}\right)_{z=L / 2} R d \theta=\varepsilon U L C
$$



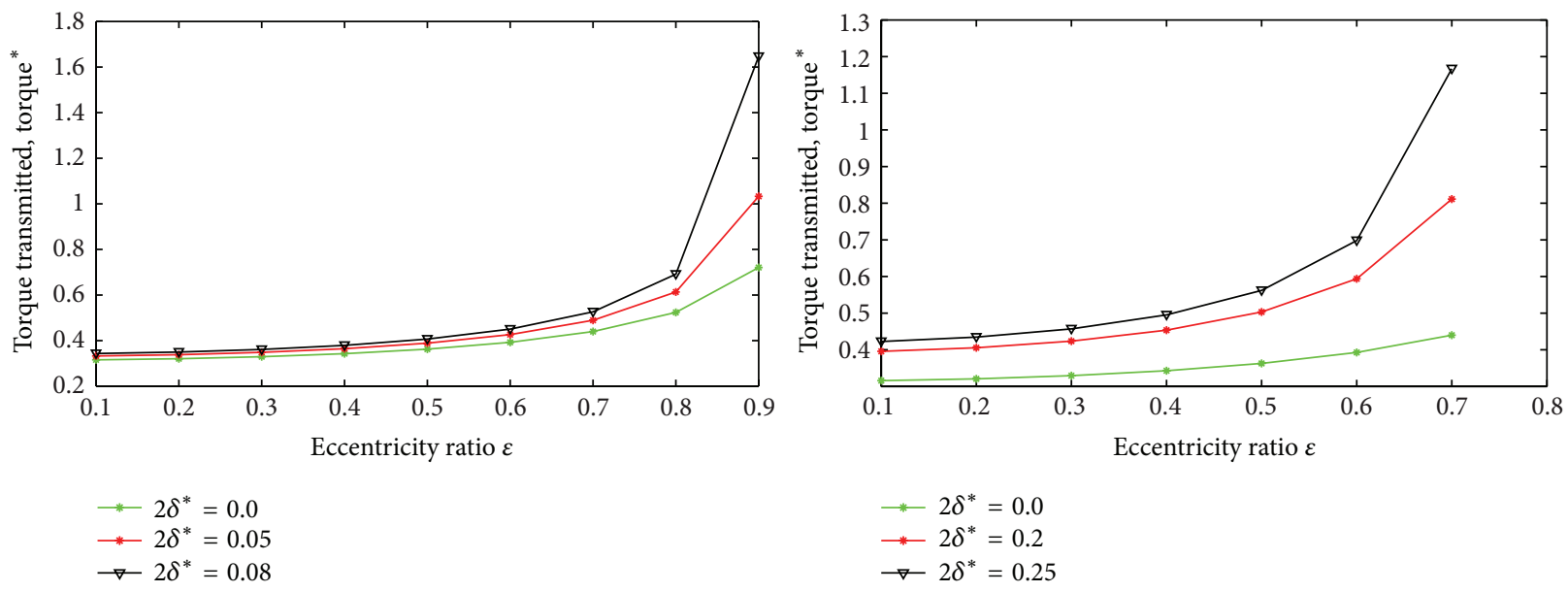

FIGURE 9: Nondimensional torque transmitted of short journal bearing as a function of the adsorbed layer thickness $\left(2 \delta^{*}\right)$.
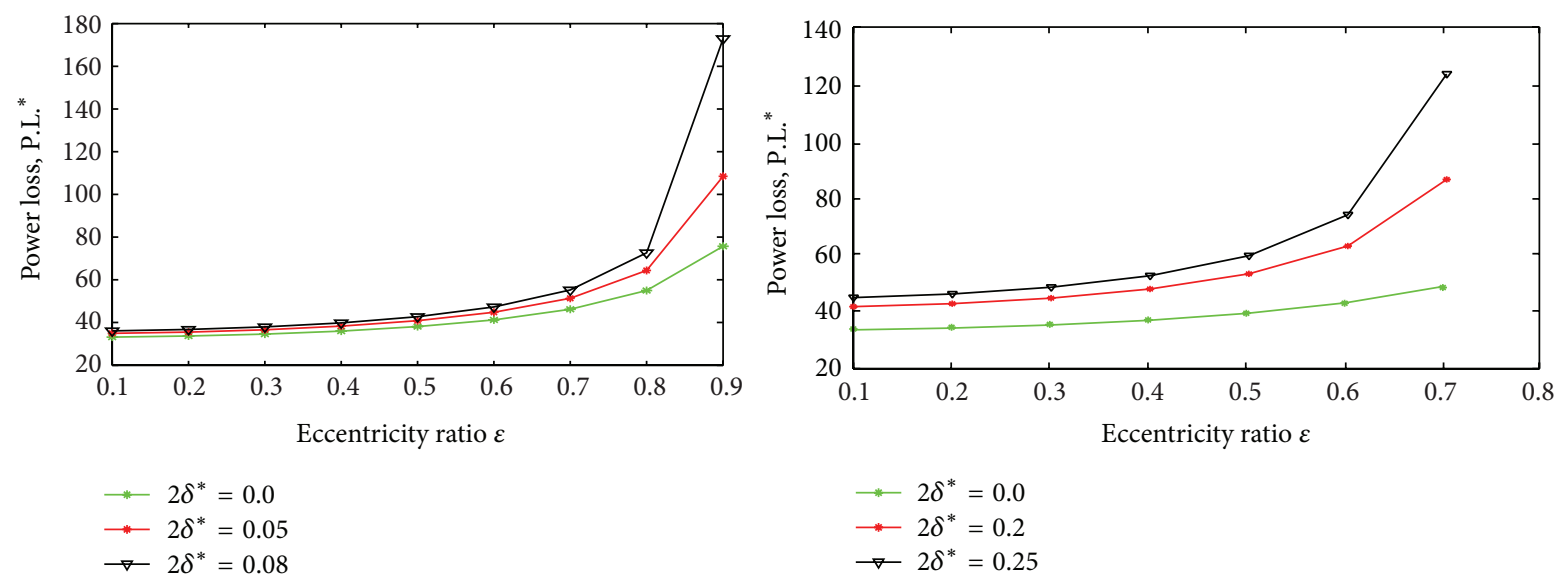

FIGURE 10: Nondimensional power-loss of short journal bearing as a function of the adsorbed layer thickness $\left(2 \delta^{*}\right)$.

During the calculation of flow rate through the short journal bearing the effect of adsorbed layer thickness $(2 \delta)$ is considered.

\section{Results and Discussion}

The nondimensional adsorbed layer thickness $\left(2 \delta^{*}\right)$ corrected equation (4) is combined with (2) to solve the thin film lubrication effect on the performance of short journal bearing. The numerical method is used here to find the solution of (7). During the calculation of different bearing parameters two cases are under consideration:

(i) $2 \delta^{*}=0$ refers to short journal bearing without considering adsorbed layer thickness;

(ii) $2 \delta^{*}=0.05$ to 0.8 refers to short journal bearing with considering adsorbed layer thickness.

Figure 2 shows the nondimensional film thickness profiles that are obtained by considering the effect of different values of nondimensional adsorbed layer thickness $\left(2 \delta^{*}\right)$. As shown in Figure 2 that when the nondimensional adsorbent layer thickness increases from 0 to 0.6 , nondimensional film thickness decreases too.

Figure 3 shows the nondimensional pressure profiles that are obtained by means of the finite difference method for the different values of nondimensional adsorbed layer thickness $\left(2 \delta^{*}\right)$. Figure 3 shows that when the nondimensional adsorbent layer thickness $\left(2 \delta^{*}\right)$ increases, nondimensional pressure also increases. The more the eccentricity, the more the pressure curve offset.

Figures 4 and 5 show the nondimensional pressure distribution in the circumferential direction of the short circular journal bearing at various eccentricity ratios $(\varepsilon=0.01$ to 0.7$)$ as a function of different values of nondimensional adsorbed layer thickness $\left(2 \delta^{*}\right)$. It shows that the dimensionless pressure $p$ increases with increment in the adsorbed layer thickness $2 \delta^{*}$ at different values of eccentricity ratio. The location of maximum pressure also changes sharply while changes in 

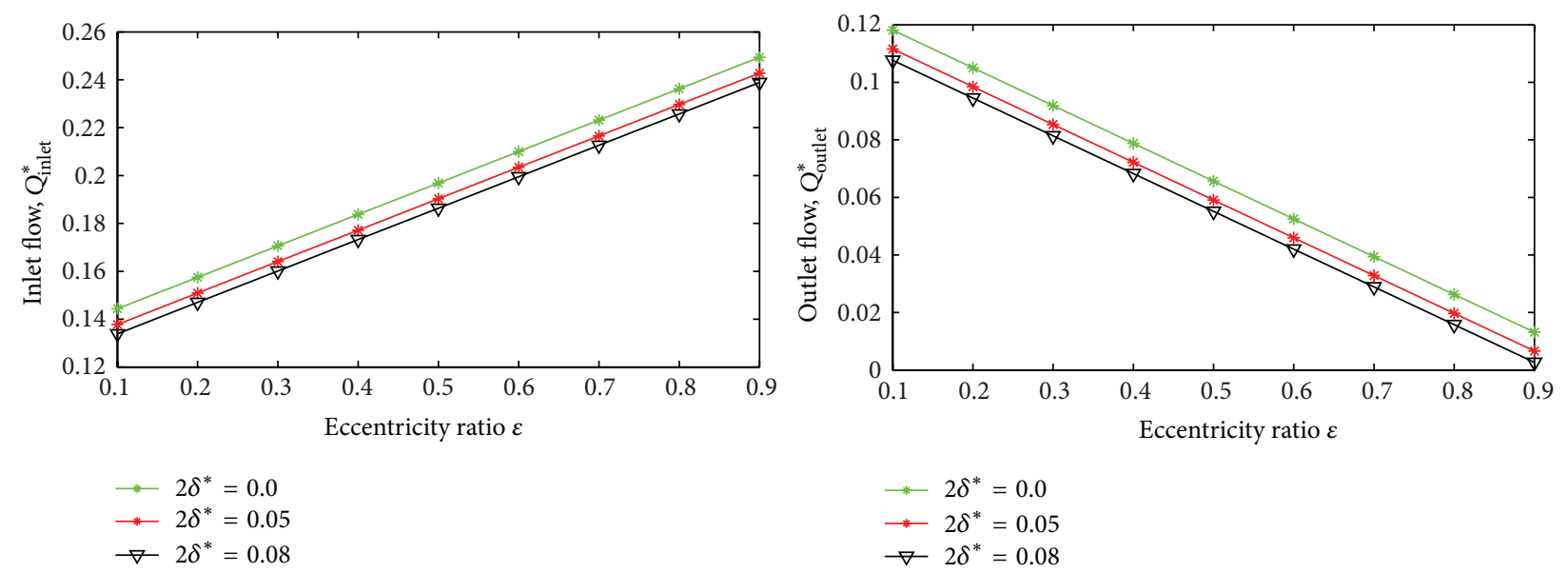

FIGURE 11: Nondimensional lubricant inlet and outlet flow of short journal bearing as a function of the adsorbed layer thickness $\left(2 \delta^{*}\right)$.

the values of the nondimensional adsorbed layer thickness $\left(2 \delta^{*}\right)$ from $2 \delta^{*}=0.0$ and $2 \delta^{*}=0.05$ and 0.25 . During the calculation the values of $\left(2 \delta^{*}+\varepsilon\right)$ are always less than 1 otherwise the lubricant will not give the proper performance under thin film lubrication. This situation requires careful selection of the values of nondimensional adsorbed layer thickness $\left(2 \delta^{*}\right)$.

The variation of the nondimensional load capacity of short journal bearing as a function of the adsorbed layer thickness $\left(2 \delta^{*}\right)$ is shown in Figure 6 . When $2 \delta^{*}$ is small, the variation of $W^{*}$ is very small as compared with $2 \delta^{*}=0$. When the value of $2 \delta^{*}$ is large, or is near to 1 , the nondimensional load capacity $W^{*}$ rapidly increases. From the figure it is clear that, when $\left(2 \delta^{*}+\varepsilon\right)$ nearer to 1 , the load carrying capacity goes towards infinity, this shows the phase transformation point of liquid film. At smaller values of $\varepsilon$ and the lower values of $2 \delta^{*}$, the change in $W^{*}$ is also very small as compared with $2 \delta^{*}=0$. In summary, the nondimensional load capacity increases with nondimensional adsorbent thickness.

Figure 7 shows the short circular journal bearing eccentricity ratio as a function of the Somerfield number and nondimensional adsorbed layer thickness $\left(2 \delta^{*}\right)$. As the eccentricity ratio increases the Somerfield number decreases sharply. Position of shaft centre is determined by using Figure 7. With the particular value of So, the steady state eccentricity ratio and attitude angle $(\varepsilon, \phi)$ is precisely obtained for different combinations of $(\varepsilon, \phi)$ and at different values of adsorbed layer thickness $\left(2 \delta^{*}\right)$. These figures give the effect of bearing parameters and adsorbed layer thickness $\left(2 \delta^{*}\right)$ for the particular bearing geometry under thin film lubrication regime.

Figures 8,9 , and 10 show the effect of nondimensional adsorbed layer thickness $\left(2 \delta^{*}\right)$ on the frictional force parameter, torque, and power-loss. At particular values of adsorbent thickness, the frictional force parameter increases as the thickness of adsorbent increases from 0 to 0.25 . When $\left(2 \delta^{*}+\varepsilon\right)$ is nearer to 1 , the frictional resistance, torque, and power-losses are increasing sharply. This shows that near the phase transformation point, the molecular forces have a sharp increase, reflecting the importance of the wall. So it is established that the friction characteristic, torque, and powerloss in conditions of thin film lubrication are quite better than traditional. In summary, as the adsorbent layer thickness increases, the frictional force parameter, torque, and powerlosses also increase as compared to the $2 \delta^{*}=0$.

Figure 11 shows the effect of nondimensional adsorbed layer thickness $\left(2 \delta^{*}\right)$ on the flow behavior of short circular journal bearing. It clearly shows that the adsorbent thickness $\left(2 \delta^{*}\right)$ affects the performance of bearing under thin film lubrication regime.

\section{Conclusion}

The effects of adsorbent layer thickness $\left(2 \delta^{*}\right)$ on the performance of short circular journal bearing are analyzed here. The various characteristics of conventional short journal bearing are compared under the effect of thin film lubrication parameters. In the regime of thin film lubrication, the value of $\left(2 \delta^{*}+\varepsilon\right)$ less than 1 must be ensured. The pressure distribution and load carrying ability of the oil film increases by the consideration of adsorbent layer thickness $\left(2 \delta^{*}\right)$. When the value of $\left(2 \delta^{*}+\varepsilon\right)$ is nearer to 1 , the location of the maximum pressure sharply changes and is very near to the point of the film export, and the film of oil lubricant will have a phase transformation. So the adsorbent layer thickness considered equation of bearing would be invalid. Strong molecular interaction is responsible for thickness of adsorbent layer and if thickness is more then it increases the load capacity and friction characteristics of the journal bearing. By considering the adsorbent action of thin film, the bearing characteristic improves the overall performance of the system.

\section{Nomenclature}

$C$ : Radial clearance ( $\mathrm{mm})$

$D$ : Diameter of journal (mm) 
L: $\quad$ Length of journal ( $\mathrm{mm})$

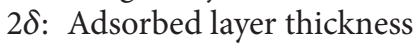

$\varepsilon: \quad$ Eccentricity ratio $=e / c$

$R:$ Radius of journal ( $\mathrm{mm}$ )

$h$ : Dimensional film thickness

$F_{f}$ : Dimensionless friction force

$T_{f}$ : Dimensionless torque transmitted

p: Dimensionless pressure

$W$ : Dimensionless load carrying capacity

$\phi$ : Attitude angle

$\theta$ : Angular coordinate in the direction of rotation

$z$ : Axial coordinate

$\mu$ : Absolute viscosity (Pa s)

$Q_{z}$ : Dimensionless lubricant flow through the bearing

So: Somerfield number.

\section{Conflict of Interests}

The authors declare that there is no conflict of interests regarding the publication of this paper.

\section{References}

[1] H. Hirani, K. Athre, and S. Biswas, "Lubricant shear thinning analysis of engine journal bearings," Tribology Transactions, vol. 44, no. 1, pp. 125-131, 2001.

[2] Q. Qingwen, Thin Film Lubrication Theory, Beijing Science Press, 2006.

[3] D. Xun, Lubrication Theory, Shanghai Jiao Tong University Press, 1984.

[4] Q. Qingwen, "The model of equivalent viscosity and test in thin film lubrication," Mechanical Science and Technology, vol. 19, no. 3, pp. 454-455, 2000.

[5] A. Harnoy, Bearing Design in Machinery-Engineering, Tribology and Lubrication, CRC Press, New York, NY, USA, 2002. 

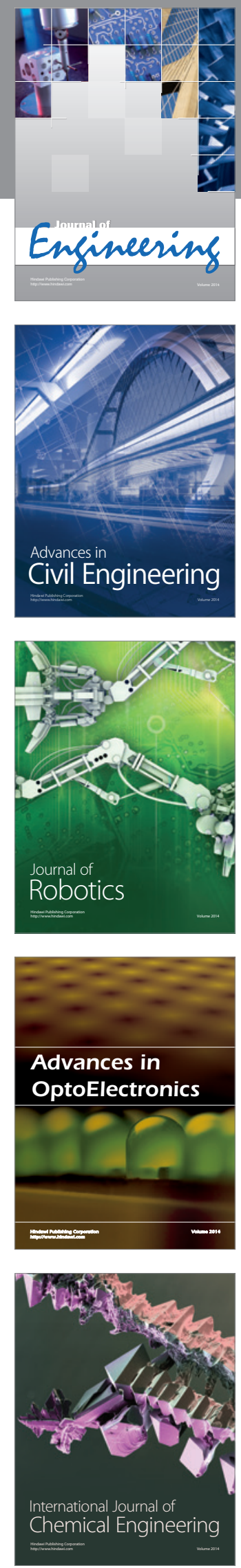

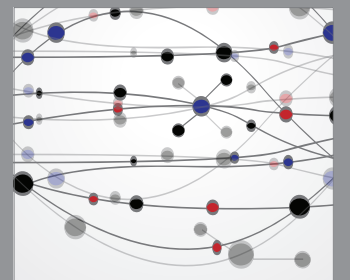

The Scientific World Journal
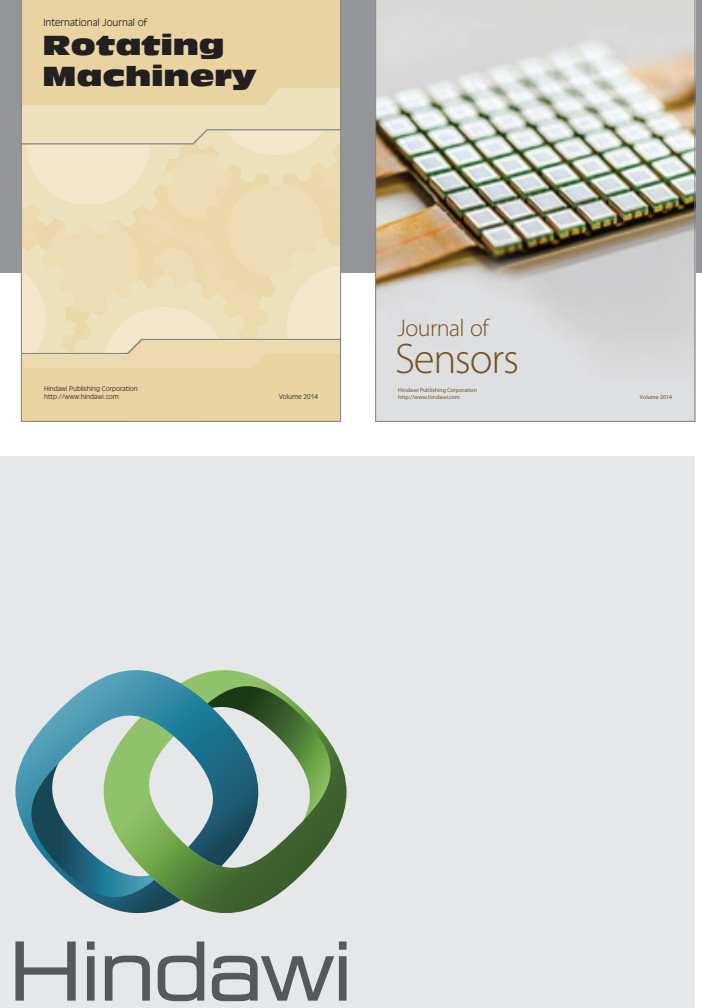

Submit your manuscripts at http://www.hindawi.com
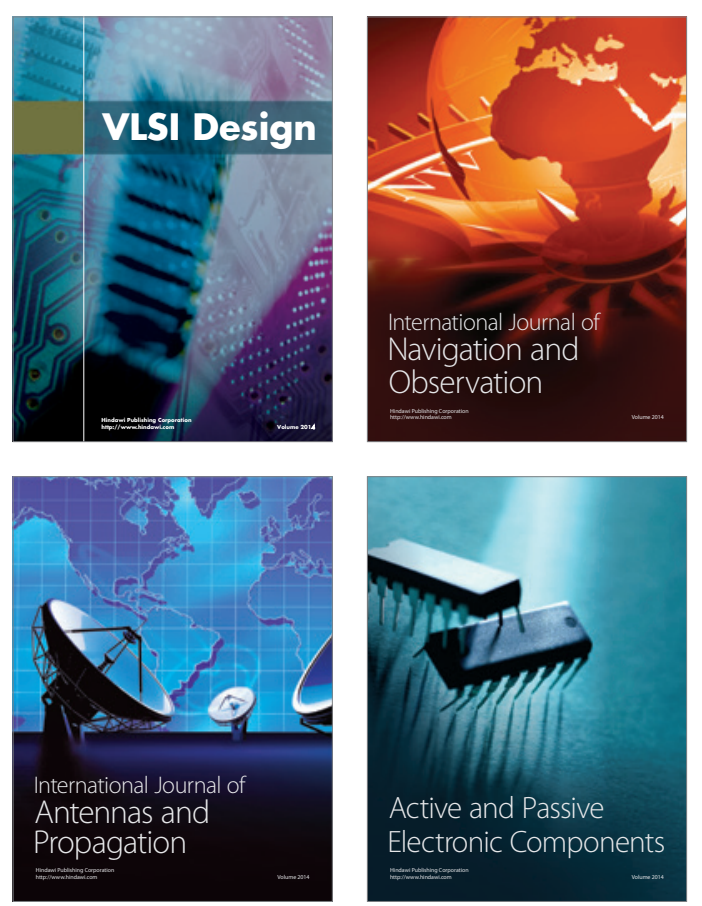
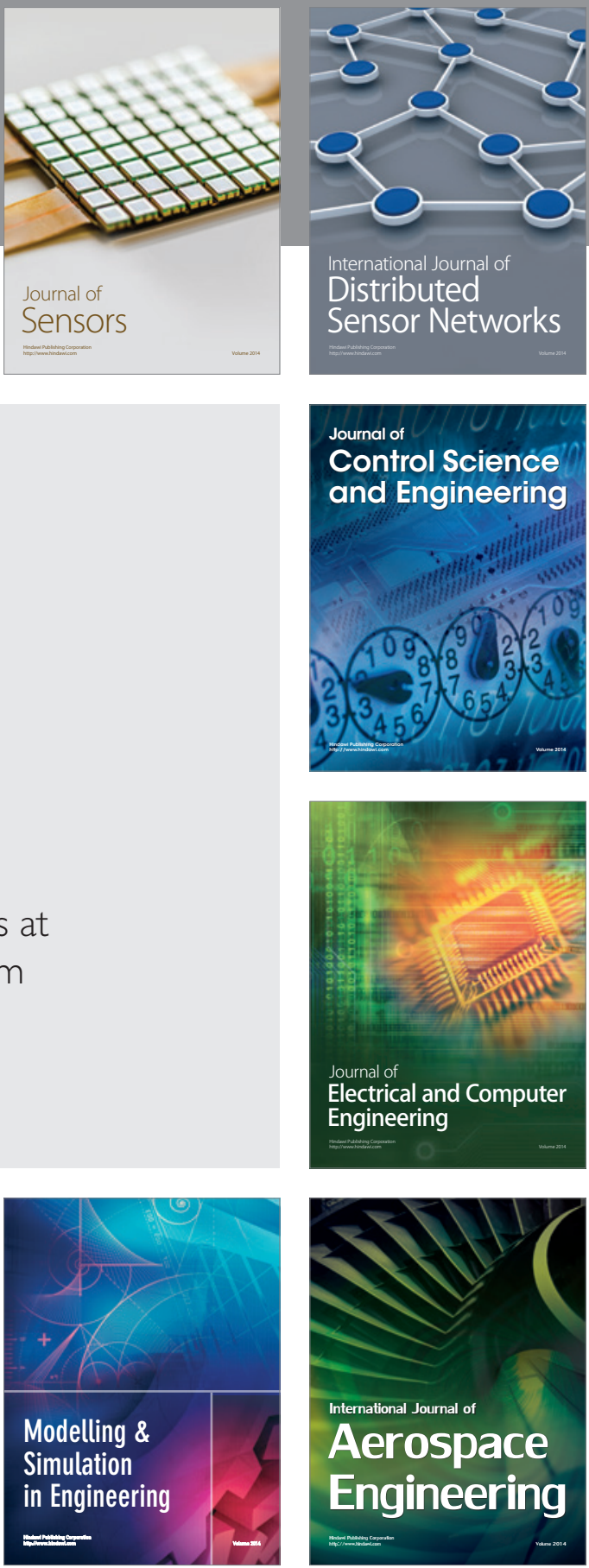

Journal of

Control Science

and Engineering
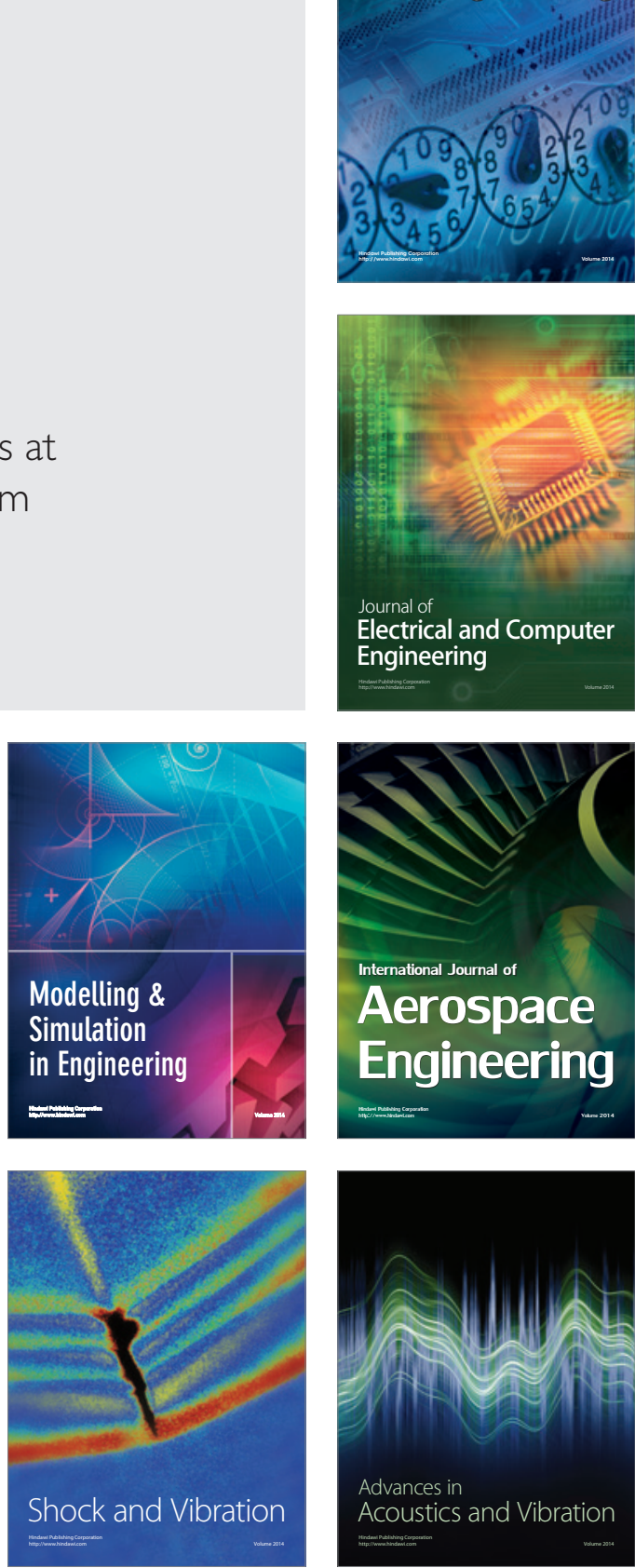